\title{
La presencia de la mujer en los filmes galardonados a lo largo de las 20 ediciones del Festival de Málaga de Cine en Español $^{*}$
}

\author{
Francisco Javier RUIZ DEL OLMO** \\ Cristina HERNANDÉZ-CARRILLO ${ }^{* * *}$
}

\begin{abstract}
Resumen
Tras los alarmantes datos publicados sobre la presencia de la mujer en el ámbito cinematográfico en España, en concreto en el sector artístico-creativo, es necesario revisar el panorama nacional, así como los grandes festivales de cine que en él acontecen. La presencia de la mujer en el cine español es un tema poco estudiado a excepción de libros como Cine y género en España o los estudios del CIMA (Asociación de Mujeres Cineastas y de Medios Audiovisuales). Es por eso que el presente artículo realiza un estudio en esa línea, analizando el número de mujeres directoras y guionistas, pretendiendo así concretar la información en el contexto del Festival de Málaga de Cine en Español, principal festival de cine de España que cuenta ya con 20 años de muestra. A través de una metodología mixta, con una base cuantitativa y una interpretación cualitativa, este artículo establece algunas líneas generales sobre las tendencias de la industria del cine y el género, con la finalidad de poner en relieve las evidentes desigualdades en las que nos vemos inmersos.
\end{abstract}

Palabras clave: Cine, Mujer, Género, Festival de Cine de Málaga, Directoras, Guionistas.

\footnotetext{
* Recibido el 06 julio 2018, aceptado el 17diciembre 2020.

** Profesor titular de la Universidad de Málaga, Facultad de Ciencias de la Comunicación, Málaga, España. firuiz@uma.es / http://orcid.org/0000-0002-1953-1798

*** Investigadora de la Universidad de Málaga, Facultad de Ciencias de la Comunicación, Málaga, España. crishcdlh@uma.es / http://orcid.org/0000-0001-5097-4661
} 
The Presence of Women in Films Awarded in the 20 Editions of The Málaga Spanish Language

Film Festival

\begin{abstract}
The publication of alarming data about the presence of women in the film industry in Spain, in particular in the artistic and creative sector, made clear the need to review the national situation, and the country's major film festivals. There is little research about the presence of women in Spanish cinema except for books such as Cine y género en España or studies by the Association of Women Filmmakers and Audiovisual Media (CIMA). For this reason, this article conducts a study in this field, analyzing the number of female directors and screenwriters. The information is specified in the context of the Malaga Spanish Language Film Festival, the main film festival in Spain, which has had 20 annual editions. Using a mixed methodology, with a quantitative and a qualitative interpretation, this article identifies some general trends about the film industry and gender to highlight the obvious inequalities found.
\end{abstract}

Keywords: Cinema, Woman, Gender, Festival de Cine De Málaga, Directors, Screenwriter. 
"Hacer cine en España es como ser torero en Islandia, dijo en una ocasión el actor y director José Sacristán. Yo añadiría que hacer cine y ser mujer en nuestro país, es torear unicornios"

Inés París, presidenta CIMA

\section{Introducción}

El cine español, así como la industria cinematográfica en general, no consigue avances significativos en cuanto a la igualdad de género. Así nos lo revelan todas las radiografías del sector realizadas a lo largo de los años. Es por eso que este estudio nace de la necesidad de visibilizar la desigualdad de género en el cine español detrás de la cámara, en concreto en los cargos que se consideran artísticos y de mayor responsabilidad: dirección y guión. Se trata de una tarea importante, ya que el cine es un espejo ideológico y la falta de mujeres en estos cargos desemboca en otros problemas, como la ausencia de personajes femeninos, o la construcción de algunos muy estereotipados. Esto, finalmente, deriva en el envío del mensaje de que las mujeres no son protagonistas en la sociedad y que además no pueden acceder estos cargos en la industria cinematográfica.

En los estudios hasta ahora consultados se observa que la presencia de la mujer en el ámbito cinematográfico es reducida. Este dato, tal y como se menciona en el Informe Anual CIMA 2015: la presencia de mujeres en el sector cinematográfico (Cuenca, 2015) resulta contradictorio respecto a lo que socialmente se presupone de los ámbitos artísticos, cuyos valores se suelen relacionar con la apertura, modernidad y progresismo, muy alejados de la brecha de género observada. Pues, se nos muestra aquí como de los 143 largometrajes estrenados en 2015 solo 29 fueron dirigidos por mujeres, siendo así un 19\% de presencia femenina, y disminuyendo en el ámbito del guión hasta el $12 \%$.

Se trata de un panorama extraño, pues como vemos en Cine y Género en España (Arranz yotros, 2010) sí disponemos de ideas y medios, habiendo un aumento de la tasa de actividad femenina española, de la feminización de las facultades de Comunicación Audiovisual y del alto nivel educativo de las mujeres españolas consumidoras de cine. Sin embargo, y en contraposición a esto, se nos ofrecen los siguientes datos: de 2000 a 2006 se realizaron 877 filmes españoles según el Instituto de la Cinematografía y de las Artes Audiovisuales (ICAA) de entre los cuales solo dirigieron un $7,4 \%$ de mujeres frente a los varones, que (excluyendo coautorías) representan un $91 \%$ del total. Otros estudios más recientes señalan también el bajo porcentaje de mujeres directoras (Del Corral, 2017); además esto no se trata de un caso singular ni único de España, sino que numerosos estudios en otros países muestran una problemática similar. En un estudio que recoge de forma enciclopédica 720 directores de cine relevantes de la cinematografía mundial los porcentajes de mujeres directoras no alcanzan el 10\% (Torres, 2008); igualmente coincide en ese bajo porcentaje en España y otros países el estudio de Merino (1999), a partir de la elaboración de un diccionario de mujeres directoras. Por todo ello cabe por lo tanto preguntarse cuáles son los obstáculos que las mujeres encuentran para no lograr la igualdad en este ámbito.

Ante estas cifras tan alarmantes, se considera imprescindible la realización de este tipo de investigaciones. La importancia de estudiar estas cuestiones de género permite conocer situaciones de desequilibrios de poder en la industria, pudiendo así entender mejor la realidad del ámbito estudiado. Además, se considera que el cine, al ser una industria cultural, es transmisor de valores a la sociedad y ayuda a la configuración de la realidad social, y por lo tanto se debe de perseguir, tal y como se hace en otros ámbitos, la igualdad.

Es cierto que a grandes rasgos los datos han mejorado, sin embargo, siguen siendo insuficientes y la industria cinematográfica continúa perpetuando trabajos estereotípicamente polarizados. Se observa así una segregación laboral significativa según el género de las personas que ejercen distintas ocupaciones profesionales, algo que nos permite detectar las desigualdades por sexo reflejadas en la jerarquía tanto vertical como horizontal.

Así lo vemos en los datos de mujeres encargadas de vestuario, maquillaje y peluquería, frente a las técnicas, ya sean de sonido, efectos especiales o cámaras. El término para señalar esta 
situación de discriminación laboral es "guetos de terciopelo", sectores laborales que se feminizan y se acaban considerando apto para mujeres (Núñez, 2010). En el estudio realizado en el capítulo Las categorías profesionales en el cine del libro Cine y género en España (Arranz y otros, 2010) se diferencia entre el Grupo Especialista (considerados como profesionales de cualificación media: peluquería, vestuario y maquillaje) y el Grupo Técnico (carácter técnico y trabajo cualificado: cámara, sonido y efectos especiales).

La raíz de esta diferenciación es más profunda, pues es evidente que en la juventud los chicos realizan (o realizaban) mayoritariamente estudios de ramas técnicas mientras que las chicas se concentran en ramas más feminizadas de peluquería y estética. A grandes rasgos se aprecia una feminización del grupo especialista, siendo en 2006 de casi un $80 \%$ de mujeres, y una masculinización del grupo técnico, siendo por el contrario de un $20 \%$. Observamos además en este capítulo de libro que predominan también los hombres en el resto de los grupos: directivo, artístico, ejecutor y técnico; siendo así mayoritarios en cuatro de cinco categorías profesionales.

En cuanto a la segregación ocupacional vertical, el estudio nos muestra datos sobre el grupo directivo-creativo (dirección y guión), que incluyen los puestos tratados en este estudio. Es en el número reducido de personas en esta posición de primer nivel donde se hace la toma de decisiones, y es aquí donde la presencia de mujeres es claramente minoritaria. Podemos ver que de 2000 a 2006 no se han producido cambios, y que la presencia de las mujeres pasa por altibajos sin definir una tendencia clara. Aún así, es destacable que las guionistas, que suelen alcanzar el $15 \%$ en casi todos los años, son superiores a las directoras (disponedoras de mayor poder en el proyecto), que no llegan a representar ni el $10 \%$ en la mayoría de los casos.

Otro aspecto curioso para destacar es el del género fílmico, pues más de la mitad de las películas dirigidas por mujeres en 2015 y 2014 son documentales, las cifras en cuanto a ficción, que es el ámbito que ocupa este trabajo, son mucho más bajas. En 2011 se estrenaron 187 largometrajes de los cuales 19 fueron dirigidos por mujeres, pero solo 4 fueron de ficción. Cabe aquí mencionar que los documentales tienen un recorrido comercial diferente y no disponen del mismo presupuesto ni aspiran a la misma recaudación, su ciclo de vida suele producirse en los festivales y pocos llegan a estrenarse en salas. Así lo confirmaba Inés París, antigua presidenta de CIMA, que hacía declaraciones a raíz de los datos de 2011, alegando que la creación femenina se había refugiado en el documental, pues tenían muy complicado el acceso a las grandes producciones, a los grandes presupuestos y festivales, por lo que buscaban otras vías.

Es por lo tanto un hecho que las mujeres se ven sometidas al denominado "techo de cristal", un término simbólico que hace referencia a la limitación de éstas al ascenso laboral y a las cúpulas directivas o cargos de mayor responsabilidad en la jerarquía empresarial. Este "techo de cristal" contemporáneo oculta una discriminación indirecta, pues no está reflejada en las leyes, pero, tal y como avalan los datos expuestos anteriormente, se manifiesta en los resultados diferenciales. Esperanza Roquero (2012) ya suscribía este hecho: «conforme más alta es la posición ocupacional, menor presencia de las mujeres mientras que, inversamente, éstas se concentran en las ocupaciones con menor grado de cualificación en el sector». Realizó un estudio en el cual se llevó a cabo un análisis de los discursos de profesionales situados en la más alta jerarquía de la cinematografía española. Los resultados obtenidos reflejan cuatro posiciones diferentes.

Por un lado, desigualdad por razón de género, que reconocen abiertamente los entrevistados aludiendo al carácter injustificado de tal desigualdad y localizando sus causas en la lógica social imperante. También se alude así a la desigualdad como norma social, culpando a la sociedad en general. Llaman más la atención los dos últimos resultados, en los cuales se afirma que viven en un entorno en el que la mujer ha alcanzado plenos derechos: la igualdad de género y la autoexclusión (achacan aquí el problema a la falta de motivación de la mujer) y la igualdad de género y carácter natural de las diferencias "innatas" entre los dos sexos (lo cual demuestra per se una actitud sexista).

La falta de reconocimiento de la mujer en el ámbito cinematográfico es algo persistente desde los inicios del propio medio. Tal y como menciona María Concepción Martínez (2008) en la trayectoria de mujeres directoras que realiza, resulta sorprendente la ausencia en los manuales de Historia del cine de la presencia de la mujer, pues la primera película con argumento es obra de una directora, la francesa Alice Guy, que filmó en 1896 El hada de las coles. El propietario de la 
productora Gaumont la autorizó a tomar la cámara al constatar que su capacidad creativa estaba por encima de su puesto como secretaria de la empresa. Además de la dirección, ella destacó en el guión, pues al ver las imágenes en movimiento de los hermanos Lumiére tuvo la idea de inventar y desarrollar historias. Encontramos que algunas de las películas más famosas de la historia del cine están escritas por mujeres, como Metrópolis (1927) por Theavon Harbou; El acorazado Potempkin (1925), que Nina Agadjanova-Shutko firmó junto con Eisenstein; o el caso de Alma Reville, que a la sombra de su marido Alfred Hitchcock escribió quince de sus películas.

A pesar de esta situación tan desventajosa, las mujeres sí que son posteriormente galardonadas y reconocidas. Así nos lo muestra Trinidad Núñez (2010) en una radiografía de 20 años de cine de directoras en este país. De entre las 56 directoras de largometrajes de ficción que la autora considera entre 1989 hasta 2009, comprendiendo 122 películas, se concluye que el 87,5\% de ellas ha obtenido alguna distinción profesional. Ella teoriza que puede ser debido a que, en esos territorios tan masculinizados, hay que ser especialmente buena para sobrevivir. Observamos aun así que las mujeres salen mejor paradas de las nominaciones, pues de las 23 ediciones de los Premios Goya sólo en 3 ocasiones una mujer ha sido premiada como Mejor Directora, otras 3 como Mejor Directora Novel y 2 ha sido premiada como Mejor Película una dirigida por una mujer. Tras estos datos se considera necesario por lo tanto analizar nuestros festivales, y tras repasar el panorama nacional en el que se ve envuelto la mujer, tanto el papel de directora como de guionista, establecer algunas líneas generales y tendencias.

Es importante resaltar que, desde hace tiempo, y con más intensidad en los últimos años, se están desarrollando algunos festivales destinados a dar visibilidad a las mujeres como autoras. Cabe aquí nombrar el Festival Internacional de Cine Realizado por Mujeres, que se celebró en Madrid en los años ochenta y noventa y contaba con el apoyo de varios organismos, como el Instituto de la Mujer o la Filmoteca Española. Hoy día contamos con el Festival Internacional de Cine y Mujer "Miradas Madrid", que se organiza entre el Ayuntamiento de Madrid y el ya citado CIMA. También hay otros festivales más reducidos como son la Muestra Internacional de Cine realizado por Mujeres, realizado por la Universidad de Zaragoza, o la Muestra de Cine Realizado por Mujeres de Bilbao (Martínez, 2008).

Más allá, el ámbito a tratar, el Festival de Málaga de Cine en Español ya está incluyendo una sección llamada "afirmando los derechos de la mujer". Tras la celebración de 20 ediciones, cuenta con un gran prestigio, siendo el festival nacional más importante de cine español, y recientemente ampliándolo a "en español" e incluyendo así películas sudamericanas. Dado que su objetivo primero es favorecer la difusión y promoción de nuestra cinematografía y servir de escaparate a material inédito, es relevante analizar qué se está premiando, y si la mujer está siendo suficientemente representada.

\section{Objetivos}

La finalidad de este trabajo es conocer la representación de la mujer en el Festival de Málaga de cine en español. Para ello se han marcado los siguientes objetivos:

- Conocer el panorama nacional de la situación de la mujer en el ámbito cinematográfico.

- Analizar el número de mujeres directoras y guionistas galardonadas a lo largo de las 20 ediciones del Festival de Málaga.

- Comparar la evolución de las directoras y las guionistas en los últimos años.

- Buscar el origen de esta problemática en base al discurso de las cineastas españolas.

\section{Metodología y muestra seleccionada}

Para ello se ha llevado a cabo una metodología mixta, pues se considera que en las ciencias sociales ambas se requieren y se complementan y dan lugar a unos resultados más completos. Se hará uso de la cuantitativa a la hora de recoger los datos sobre cada año de festival, catalogando posteriormente las películas galardonadas y estableciendo en cada una el número de hombres y mujeres que llevan a cabo la dirección y el guión. Esto dará lugar a diferentes porcentajes (número 
de mujeres por año, número de directoras totales, de guionistas...) que serán interpretados y analizados en relación con el panorama nacional y a la revisión bibliográfica de manera cualitativa.

La selección de la muestra se ha acotado a cuatro galardones que entrega este festival:

- Biznaga de Oro o premio a Mejor Película.

- Biznaga de Plata a la Mejor dirección.

- Premio especial de jurado.

-Premio del público.

Se ha optado por esta selección porque se observa que son los únicos premios que han prevalecido a lo largo de las 20 ediciones de festival, aunque hayan cambiado en algún momento su nomenclatura, por ejemplo, en la primera edición lo que se llamó "primer premio" en la última se considera "biznaga de oro". Al seleccionar esta muestra se puede establecer fácilmente una temporalidad para llegar a conclusiones más exactas. También por el hecho de que estos cuatro galardones son considerados los más importantes de cara a lo que se va a analizar. Además, se encuentra variedad de la elección de los ganadores, siendo unos elegidos por el jurado especializado y otros por el público. En estas cuatro categorías se ven comprendidas aquellas películas que se observaron que en un conjunto tenían mejor dirección y guión. Cabe también mencionar que en las estadísticas de cada año se ha optado por no repetir películas a la hora de contar los datos en el caso de que fueran galardonadas en los premios coetáneos, para así poder obtener unos resultados más fidedignos.

Por otra parte, se ha elegido analizar únicamente el papel de las mujeres directoras y guionistas porque encarnan las profesiones de más poder y toma de decisiones dentro del ámbito cinematográfico. Pertenecería también a este lugar la productora, pero se ha preferido escoger los puestos que también representan una parte creativa, relacionadas con la toma de decisiones específicamente en cuanto a argumento, guión técnico, dirección de actores... y menos relacionado con el ámbito económico. Se usa en la bibliografía consultada la terminología para estas dos profesiones nombradas como "grupo directivo-creativo" (Arranz y otros, 2010).

\section{Contenido}

\subsection{Evolución a lo largo de los años}

Es necesario presentar en primer lugar los resultados obtenidos a raíz de la catalogación de todas las películas ganadoras en los cuatro premios seleccionados (mejor película, mejor dirección, premio del jurado y premio del público) desde 1998 hasta 2017. A lo largo de este periodo se van estableciendo porcentajes, viendo así en cada año cuál es la participación de las mujeres con respecto a los hombres. 
Figura 1. Evolución del porcentaje de mujeres directoras y guionistas en el

Festival de Málaga de Cine en Español.

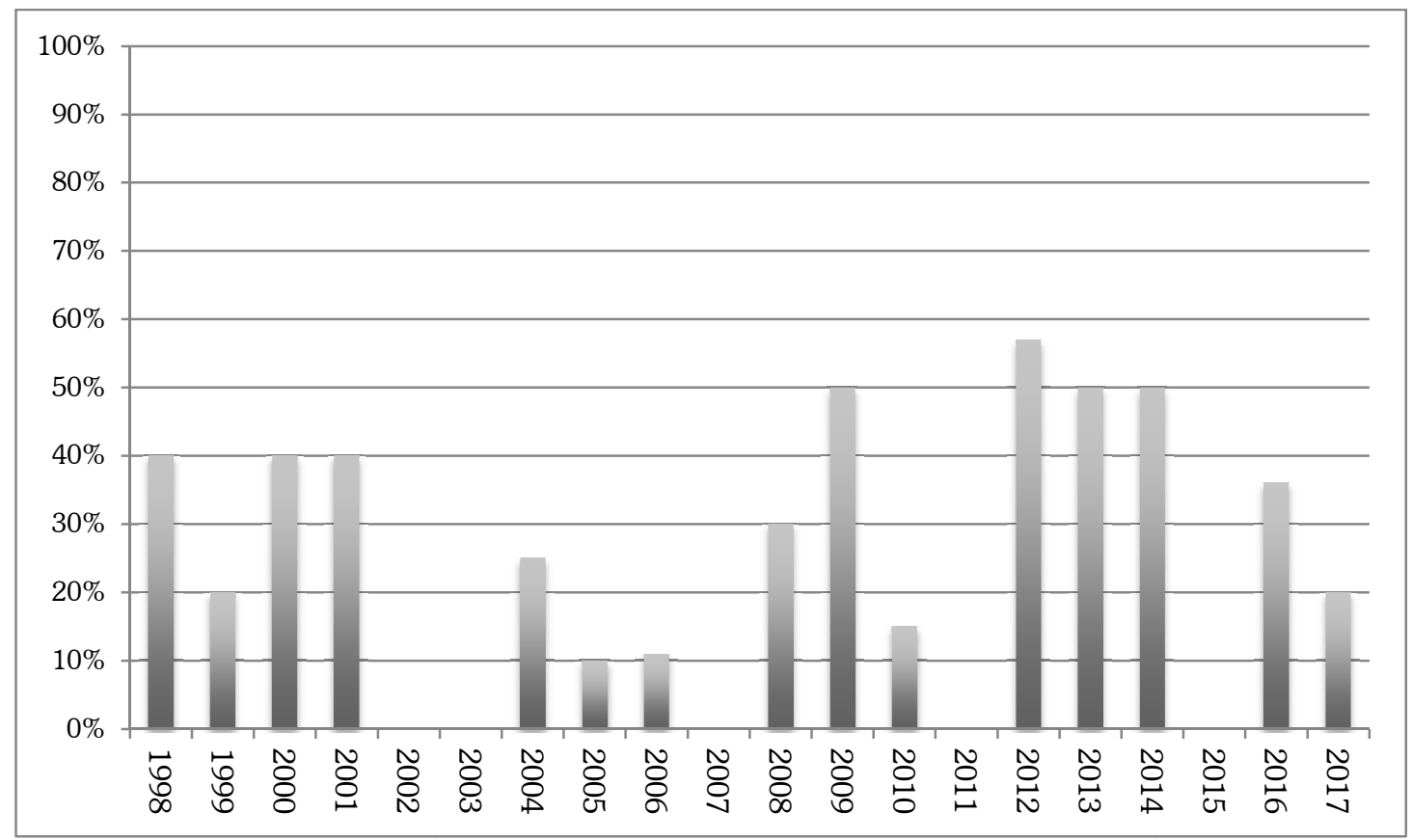

Fuente: Elaboración propia a partir de la base de datos del Ministerio de Educación, Cultura y Deporte.

Vemos en este diagrama de barras cómo a pesar del paso de los años no hay un avance claro y no se pueden establecer tendencias determinadas hacia un crecimiento continuo. Lo esperado sería que, conforme avanza la sociedad y la mujer se va viendo integrada en más ámbitos laborales en persecución de la igualdad, se observara un aumento con el paso del tiempo, algo que no vemos en estos datos.

Así pues, es necesario remarcar lo obvio, que la presencia de mujeres en estos cargos no es igual que la de los hombres. Y reivindicar que, aunque los datos nos puedan parecer "buenos" en comparación con los que estamos acostumbrados a recibir del cine, no son suficientes. En las películas analizadas se cuenta con un equipo total de 181 personas, entre mujeres y hombres, directoras/es y guionistas; de este total tan solo 46 son mujeres frente a los 135 hombres. El dato resultante es que entre directoras y guionistas hay una presencia del $25 \%$ de mujeres en los cuatro premios seleccionados a lo largo de las 20 ediciones del Festival de Málaga de Cine en Español.

Es además significativo, y merece ser mencionado, el hecho de que durante cinco años (2002, 2003, 2007, 2011 y 2015), que además están dispersos en la línea temporal, no haya ninguna mujer guionista ni directora en las películas premiadas. Esto en algunos casos se debe a que ese año una única película se llevó varios galardones, como ocurre en 2002 con El otro lado de la cama. Sin embargo, en otros como 2011 hay cuatro películas diferentes (Cinco metros cuadrados, ¿Para qué sirve un oso?, Catalunya überalles! y Amigos) y en ninguna de ella participó una mujer en dirección o guión.

A pesar de que los datos son en estos años catastróficos, también es cierto que durante otros el porcentaje es mayor que en el panorama global nacional, siendo estos en algunos casos del $50 \%$ (alcanzando la igualdad entre hombres y mujeres) y en 2012 incluso del 57\% (gracias a películas hechas enteramente por mujeres en los cargos analizados como Els nens salvatges). Esto confirma lo nombrado por otros autores como Núñez (2010), que observa que las mujeres son reconocidas y galardonas a pesar de ser menos numerosas en esta profesión que los hombres.

A lo largo de la revisión bibliográfica hemos visto cómo las mujeres encuentran más dificultad a la hora de encontrar financiación y es por eso que, muchas veces, se alojan en los documentales, sin poder acceder a la ficción. Podría ocurrir que el Festival de Málaga, al ser un escaparate para películas independientes, dé más oportunidades a aquellas obras "de autor" (o "de autora" en este caso) alejadas de las grandes superproducciones, lo cual viendo el gran número de mujeres que se 
tienen que producir sus propias películas por problemas de financiación, puede favorecer. O por lo menos en comparación con otros premios, como los Goya, en los que se compite con películas de gran presupuesto y que siguen otros patrones.

Para ilustrar lo nombrado anteriormente podríamos poner como ejemplo las últimas ediciones de los Goya, en las que las películas más independientes (como puede ser Tarde para la ira en ese ámbito) acumulan menos estatuillas en comparación con superproducciones (Un monstruo viene a verme). Remarcar también que se observa últimamente una tendencia de películas ganadoras en el Festival de Málaga que son posteriormente galardonadas con un Goya a la Mejor dirección Novel (es el caso de A cambio de nada, 10.000 KM, Tres dies amb la familia, Azul Oscuro Casi Negro...), demostrando así que este festival sí que cumple su función de dar a conocer nuevos talentos del ámbito cinematográfico.

En cualquier caso, el panorama del Festival de Cine de Málaga acumula mejores datos en cuanto a la presencia de la mujer que los premios Goya, pues en estas 23 ediciones solo 2 una mujer ha sido premiada a mejor película siendo ella directora, y 3 a mejor dirección, siendo un total de 5. La primera en ganar el Goya a la Mejor Dirección fue Pilar Miró con El perro del hortelano en 1996, seguida de Icíar Bollaín en 2003 con Te doy mis ojos (ganadora también a mejor película), por último, en 2005 Isabel Coixet con La vida secreta de las palabras (la segunda y última premia a mejor película).

Seleccionando los mismos tipos de premios y en 20 ediciones en los datos del Festival de Málaga podemos observar que 5 mujeres han dirigido filmes premiadas como mejores películas y otras 5 han sido galardonadas como mejor directoras. Además, desde 2004 se incluye en este festival la sección Zonazine, que se inaugura para dar cabida a películas más independientes, y abre así espacio para más cineastas.

\subsection{Entre directoras y guionistas}

Cuando separamos los datos en función de las categorías seleccionadas se puede observar que hay una gran diferencia entre el porcentaje de mujeres directoras y guionistas. Siendo siempre menos numerosas las primeras.

Tabla 1. Número total de mujeres directoras en comparación con mujeres guionistas en el Festival de Málaga de Cine en Español.

\begin{tabular}{|c|c|c|c|c|c|c|c|c|}
\cline { 2 - 9 } \multicolumn{1}{c|}{} & \multicolumn{2}{c|}{ Mejor Película } & \multicolumn{2}{c|}{ Mejor Dirección } & \multicolumn{2}{c|}{ Premio Jurado } & \multicolumn{2}{c|}{ Premio Público } \\
\cline { 2 - 10 } \multicolumn{1}{c|}{} & Director/a & Guionista & Director/a & Guionista & Director/a & Guionista & Director/a & Guionista \\
\hline Hombres & 16 & 21 & 16 & 18 & 15 & 26 & 21 & 26 \\
\hline Mujeres & 5 & 13 & 5 & 12 & 4 & 5 & 3 & 7 \\
\hline Total H+M & 21 & 34 & 21 & 30 & 19 & 31 & 24 & 33 \\
\hline $\begin{array}{c}\text { Total \% } \\
\text { Mujeres }\end{array}$ & $24 \%$ & $38 \%$ & $24 \%$ & $40 \%$ & $21 \%$ & $16 \%$ & $12 \%$ & $21 \%$ \\
\hline
\end{tabular}

Fuente: Elaboración propia a partir de la base de datos del Ministerio de Educación, Cultura y Deporte.

A lo largo de las veinte ediciones del festival los datos de las guionistas galardonadas oscilan entre el $40 \%$ y el $16 \%$, mientras que las directoras tienen una media más baja, siempre alrededor del $20 \%$, llegando hasta a alcanzar el $12 \%$. A la hora de hacer la media los datos son los siguientes: el número total de directoras premiadas en la muestra seleccionada en las 20 ediciones es aproximadamente del $20 \%$, mientras que las guionistas son del $30 \%$.

Comparando estos datos con la tendencia del panorama nacional observamos algunos puntos en común. En el estudio encontrado en Cine y género en España (Arranz y otros, 2010) vemos la evolución de las categorías profesionales de las mujeres de 2000 a 2006. De cara a poder establecer paralelismos se ha realizado la misma tabla con los datos obtenidos del festival analizado. Los 
resultados son diferentes, aunque nos hacen llegar a las mismas conclusiones. El puesto de directora es más difícil de alcanzar que el de guionista, pues se considera que es más alta su jerarquía de poder, entra aquí en juego el ya definido "techo de cristal", es una profesión altamente masculinizada.

Observamos que en la gráfica de los datos del festival hay un "alto" número de mujeres en comparación con el escaso número de ellas que presentan películas, esto se debe a lo ya nombrado anteriormente, sus trabajos suelen ser reconocidos a pesar de ser minoritarios. El gráfico extraído del libro Cine y género nos presenta unos datos más lineales, quizás debido a que la muestra en más grande, aunque tal y como dice Arranz (2010) nos encontramos con una tendencia poco clara e incierta. No vemos signos de evolución alguna. En el Festival de Málaga nos encontramos con la ausencia de directoras en las películas premiadas en 2002, 2003, 2005, 2006 y 2007. Si comparamos esto con los datos de esos años no es de extrañar, pues el porcentaje total de ellas en el panorama nacional ni siquiera supera el 12\%, quedándose incluso en 2006 alrededor del 5\%, unos datos alarmantes. Es por lo tanto coherente con respecto a la industria que, de tan pocas películas hechas por mujeres, ninguna llegara al festival.

Figura 2. Evolución de presencia porcentual de mujeres del grupo directivo-creativo en el panorama nacional de 2000 a 2006.

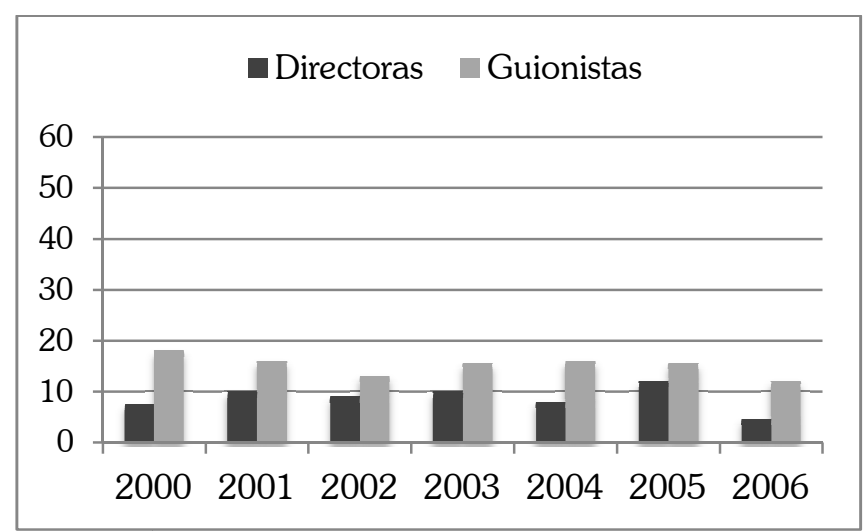

Fuente: Extraído de Cine y género en España (Arranz y otros., 2010) ${ }^{1}$

Figura 3. Evolución de presencia porcentual de mujeres del grupo directivo-creativo en el Festival de Málaga de Cine en Español de 2000 a 2006.

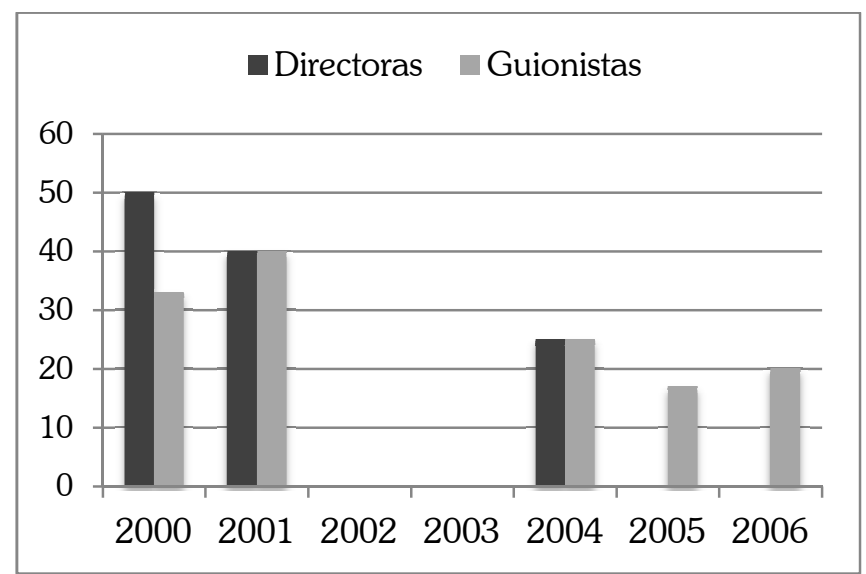

Fuente: Elaboración propia a partir de la base de datos del Ministerio de Educación, Cultura y Deporte.

\footnotetext{
1 Estudio realizado por Esperanza Roquero (Arranz y otros, 2010) con la información de la base de datos de Anuarios del Cine, publicados por el Instituto de la Cinematografía y de las Artes Audiovisuales (ICAA) (Ministerio de Cultura).
} 
A raíz de estas gráficas y otros resultados, no sorprende que Inés París y demás cineastas del CIMA nos hablen del "misterioso caso de las directoras inexistentes" y especulen, buscando posibles razones para este hecho. Cabe aquí hacer mención de algunas de ellas para así ponerlas en relieve y poder atacar el lastre de la desigualdad en este ámbito.

Para empezar, ella hace referencia a que las guionistas sean más numerosas que las directoras por cuestiones de tendencia histórica. Señala que a lo largo de la historia la escritura ha sido una técnica relativamente accesible a mujeres, aunque fuera de manera anónima y oculta. Se podía realizar con pocos medios y aquellas que tenían la suerte conforme avanzaban los siglos de aprender a leer y a escribir se aventuraban a hacerlo.

En cuanto a las directoras, esboza algunas características atribuidas a la dirección cinematográfica. Para contextualizar, determina que se trata de un medio masculinizado (como pueden confirmar todos los datos arrojados anteriormente), donde las mujeres se ven obligadas a convencer a los demás de su capacidad para afrontar la dirección siempre ante un auditorio mayoritariamente masculino. Pues ya hemos visto que, a excepción de vestuario, maquillaje y peluquería, el resto de los sectores están dominados por el otro género, sobre todo en los cargos que se ostenta más poder: productores, distribuidores y directivos. A esto se añade que a veces la temática propuesta o el tratamiento les sorprende, incomoda, o directamente lo consideran desconocido o poco interesante.

Dirigir presupone grandes habilidades de creación y liderazgo, así como dominar muchos aspectos diferentes tanto técnicos como creativos (fotografía, dirección de actores, montaje...). A raíz de su propia experiencia personal señala que también influye la diferencia de personalidad de las directoras, posiblemente a raíz de los objetivos vitales inculcados en la educación: "las mujeres aún no somos educadas y potenciadas en la asertividad, la fe ciega en una misma, el 'egoísmo', y la cabezonería que hacen falta para sobrevivir en esta profesión" (Arranz y otros, 2010:371). Añade que, a lo largo de su carrera, en numerosas exposiciones de cortos y otros proyectos ha visto mujeres con talento sobresaliente y gran personalidad y que se muestran sorprendidas ante la acogida de sus trabajos. Ella opina que su inteligencia y sensibilidad las puede llevar a ser especialmente autocríticas.

\subsection{Discusión de la problemática}

Esta situación que se ha descrito anteriormente debe mejorarse, en primer lugar, a través del conocimiento de lo que está sucediendo: arrojar datos y que la sociedad los reciba para así poder reflexionar acerca de ellos. Es cada vez más común que las cineastas españolas lleven a cabo esta labor, denunciando su presencia minoritaria en el sector.

Así lo vemos en las entrevistas que realiza el periódico El Mundo (2017) con motivo del día de la mujer a las directoras españolas. Comienza hablando Nely Regueras, nominada al Goya a la Mejor Dirección por su primer filme María (y los demás):

\footnotetext{
Es cierto que las mujeres no tienen las mismas oportunidades que los hombres en la mayoría de disciplinas, pero no es el único factor. Tiene que ver con el país, con que durante mucho tiempo no hubiese mujeres que se plantearan a hacer cine. Quizá sus expectativas eran más reducidas por cuestiones de educación y sociales, aunque hace tiempo que la situación está cambiando. Hay que creérselo.
}

Ella alude a un factor que Inés París ya mencionaba, subyacente a la industria en sí, se trata de la educación y la sociedad. Leticia Dolera, la directora de Requisitos para ser una persona normal culpa al hecho de que no haya prácticamente referentes femeninos, pues eso hace que las chicas jóvenes se sientan incapaces de alcanzar puestos de dirección. Además, afirma que «siempre se desconfía más de una historia que va a contar una mujer», no por falta de interés del público sino por menos inversión. Paula Ortiz, directora de La Novia, hace referencia a la dificultad de la mujer para acceder a los mismos recursos que los hombres: "Mientras sigan en las mismas manos, con una concepción muy restrictiva, será muy difícil salir adelante. Hay que reivindicar la capacidad de las mujeres para sacar adelante grandes proyectos". 
Son varias voces las que mencionan la importancia de los referentes femeninos, como la de Icíar Bollaín en su entrevista para Aisge, en la que recuerda el momento en el que quiso ser directora. Fue cuando trabajó con Chus Gutiérrez, pues vio a una mujer como ella, casi de la misma edad, haciendo una película sobra mujeres, se sintió identificada con alguien cercano y se dijo a sí misma que podría dirigir cine. Habla también de la importancia de la presencia de mujeres en otras categorías profesionales del cine:

Faltan directoras, pero faltan directoras de festivales, faltan críticas de cine. Hay muchas, pero muy pocas que sean la crítica estrella del periódico. Me faltan mujeres en todos los comités que se deciden los proyectos que se hacen. (...) Necesitamos más presencia femenina entre las personas que deciden qué se hace y quién lo hace (Crespo, 2017).

Cabe mencionar que estas inquietudes y conclusiones de las directoras españolas no han avanzado con el tiempo, al igual que no lo han hecho los datos. Así lo recoge Camí-Vela (2001) en sus entrevistas a directoras de los noventa, pues llama la atención que muchas denuncian y mencionan lo mismo que en 2017, como lo hace Icíar Bollaín, nombrada anteriormente.

Otro tema recurrente y que preocupa a las directoras de los noventa es el catalogar este tipo de cine de forma diferente al cine general. Están en contra del término "cine de mujeres", al igual que directoras de hoy día. Leticia Dolera (2017) nos explica su experiencia, confirmando que muchos productores rechazaron su película alegando que "ya tenían una peli de mujeres". Encuentran estas profesionales en su mayoría que esa etiqueta les puede perjudicar, siendo motivo para acotar su público, su financiación y finalmente sus posibilidades de sacar adelante un proyecto.

Al fin y al cabo, aunque aborden temas diferentes, lo que todas denuncian es una desigualdad de condiciones a la hora de hacer una película. Concluyen que, en lo que a los galardones respecta, es algo simple: las mujeres ganan menos premios porque tienen menos oportunidades que los hombres para hacer películas.

La Asociación de Mujeres Cineastas y de Medios Audiovisuales (CIMA) está luchando activamente para acabar con esta desigualdad. Un ejemplo sería su desarrollo de propuesta de ley similar a la de otros países, como Suecia donde se reserva un $50 \%$ del presupuesto para las mujeres en la labor de producción y dirección. Esta medida ha conseguido que en ese país el porcentaje de largometrajes dirigidos por mujeres pase del $26 \%$ al $50 \%$ en cinco años.

Gracias a estos organismos y al propio público podremos evolucionar hacia un modelo más igualitario. Los espectadores van poco a poco siendo conscientes de estas circunstancias y demandan un cine diferente al que se nos ha venido ofreciendo hasta ahora. En el ámbito estudiado, el Festival de Málaga de Cine en Español en 2017, se han visto ganadoras en la Sección Oficial y en la Zonazine dos películas dirigidas por mujeres y con un argumento que versa sobre una protagonista femenina, es el caso de Carla Simón con Verano 1993 y Elena Martín con Julia Ist. Cabe por lo tanto aguardar cierta esperanza y ser críticos a la par que optimistas con este tema.

\section{Conclusiones}

Las conclusiones extraídas de este trabajo son numerosas. Por un lado, ante los datos analizados, podemos decir que el panorama nacional cinematográfico no es igualitario y que la mujer ocupa porcentajes muy bajos en cuanto a los altos cargos creativos de dirección y de guión.

Por otra parte, su presencia en el Festival de Málaga de Cine en Español es igualmente minoritaria, debido a la escasez de mujeres directoras y guionistas que hay es más difícil que sean premiadas. Aun así, es necesario decir que reciben galardones y que su trabajo se ve recompensando en un porcentaje bastante elevado.

Además, decir que en este festival analizado encontramos una presencia mayor de ellas que en otros galardones, como es el de los premios Goya. Los datos y los testimonios de las propias directoras y guionistas avalan que ellas lo tienen más difícil a la hora de conseguir financiación y apoyo a sus proyectos, y eso puede hacer que encuentren en este festival una forma de enseñar sus filmes, al contar con secciones más independientes y no competir con superproducciones de mucho presupuesto. 
También, a la hora de separar los dos trabajos analizados encontramos que las directoras ven más trabas a la hora de ejercer su profesión y ser posteriormente reconocidas que las guionistas. Esto se debe a que las directoras ejercen una función de más poder y de toma de decisiones, lo cual pertenece a un mundo muy "masculinizado". A parte de eso deben exponer sus proyectos ante otros hombres de la industria (productores, distribuidores...) que no siempre entienden sus proyectos y que los tachan de "películas de mujeres".

Por último, decir que ante esta situación sería necesario promover más medidas eficaces que acabarán con el gran sesgo de género de esta profesión, pues la cultura es la vía de comunicación a la sociedad. Es muy importante que haya más mujeres transmitiendo cultura pues así habrá más puntos de vista diferentes, más protagonistas femeninas y menos estereotipadas, además de más referentes para las futuras generaciones.

\section{Referencias bibliograficas}

ARAT, Yesim. Religion, Politics and Gender Equality in Turkey: implications of a democratic paradox? Third World Quarterly 31 (6), 2010, pp.869-884.

ARRANZ, Fátima y otros. Cine y género en España: una investigación empírica. Madrid, Ediciones Cátedra, 2010.

BILGIÇ, Ali. Turkey, Power and the West - Gendered International Relations and Foreign Policy. Londres, I.B. Tauris, 2016.

BUTLER, Judith. Corpos em aliança e a política das ruas. Rio de Janeiro, Civilização Brasileira, 2018.

CAMÍ-VELA, María. Mujeres detrás de la cámara: Entrevistas con cineastas españolas de la década de los 90. Madrid, España, Ocho y Medio. Libros de Cine, 2001.

CAMPBELL, David. Writing Security: United States Foreign Policy and the Politics of Identity, Minneapolis, University of Minnesota Press.

CRESPO, Irene. No solo faltan directoras. Faltan mujeres en toda la profesión. Aisge, 8 de marzo de 2017 [http://www.aisge.es/iciar-bollain - acceso el 01 de julio de 2018].

CUENCA, Sara. Informe Anual CIMA 2015: la presencia de mujeres en el sector cinematográfico. 2015 [http://cimamentoring.com/informe-anual-2015/ - acceso el 01 de julio de 2018].

DEL CORRAL, Pedro. Imagina ser directora de cine. Sólo un 20\% de ellas lo consigue. El Mundo, 8 de marzo de 2017 [http://www.elmundo.es/cultura/2017/03/08/58bf09c646163ff5208b4576.html - acceso el 01 de julio de 2018].

DOLERA, Leticia. Que no te engañen, el cine no tiene género. El Diario.es, 12 de marzo de 2017 [http://www.eldiario.es/tribunaabierta/Cine-mujeres-leticia_dolera-machismo-genero_6_621597844.html acceso el 01 de julio de 2018].

MARTÍNEZ, María Concepción. Mujeres al otro lado de la cámara: (¿dónde están las directoras de cine?). Espacio Tiempo y Forma. Serie VII, Historia del Arte. Madrid, 2008, pp.315-340.

MERINO, Azucena. Diccionario de mujeres directoras. Madrid, Ediciones JC, 1999.

MINISTERIO de Educación, Cultura y Deporte. Base de datos de películas de cine [http://www.mecd.gob.es/bbddpeliculas/cargarFiltro.do?layout=bbddpeliculas\&cache =init\&language $=$ es acceso el 01 de julio de 2018].

NÚÑEZ, Trinidad. Mujeres directoras de cine: un reto, una esperanza. Pixel-Bit. Revista de Medios y Educación. España, 2010, pp.121-133.

ROQUERO, Esperanza. El "techo de cristal" en la dirección cinematográfica: discursos y representaciones sociales en un caso de segregación ocupacional. Mediaciones Sociales, Madrid, 2012, pp.37-61. doi: http://dx.doi.org/10.5209/rev_MESO.2012.n10.39681.

TORRES, Augusto M. 720 directores de cine. Barcelona, Ariel. 2008. 